\title{
Establishment of Hertwig's Epithelial Root Sheath/ Epithelial Rests of Malassez Cell Line from Human Periodontium
}

\author{
Hyun Nam ${ }^{1,5}$, Ji-Hye Kim ${ }^{1}$, Jae-Won Kim ${ }^{1}$, Byoung-Moo Seo ${ }^{2}$, Joo-Cheol Park ${ }^{3}$, Jung-Wook Kim ${ }^{4}$, \\ and Gene Lee ${ }^{1, *}$
}

\begin{abstract}
Human Hertwig's epithelial root sheath/epithelial rests of Malassez (HERS/ERM) cells are epithelial remnants of teeth residing in the periodontium. Although the functional roles of HERS/ERM cells have yet to be elucidated, they are a unique epithelial cell population in adult teeth and are reported to have stem cell characteristics. Therefore, HERS/ ERM cells might play a role as an epithelial component for the repair or regeneration of dental hard tissues; however, they are very rare population in periodontium and the primary isolation of them is considered to be difficult. To overcome these problems, we immortalized primary HERS/ ERM cells isolated from human periodontium using SV40 large T antigen (SV40 LT) and performed a characterization of the immortalized cell line. Primary HERS/ERM cells could not be maintained for more than 6 passages; however, immortalized HERS/ERM cells were maintained for more than 20 passages. There were no differences in the morphological and immunophenotypic characteristics of HERS/ERM cells and immortalized HERS/ERM cells. The expression of epithelial stem cell and embryonic stem cell markers was maintained in immortalized HERS/ERM cells. Moreover, immortalized HERS/ERM cells could acquire mesenchymal phenotypes through the epithelial-mesenchymal transition via TGF- $\beta 1$. In conclusion, we established an immortalized human HERS/ERM cell line with SV40 LT and expect this cell line to contribute to the understanding of the functional roles of HERS/ERM cells and the tissue engineering of teeth.
\end{abstract}

\footnotetext{
${ }^{1}$ Laboratory of Molecular Genetics, Dental Research Institute, School of Dentistry, ${ }^{2}$ Department of Oral and Maxillofacial Surgery, ${ }^{3}$ Department of Oral Histology-Developmental Biology, ${ }^{4}$ Department of Pediatric Dentistry, Seoul National University, Seoul 110-749, Korea, ${ }^{5}$ Present address: Samsung Medical Center, School of Medicine, Sungkyunkwan University, Seoul 135-710, Korea

*Correspondence: genelee@snu.ac.kr
}

Received 5 June, 2014; revised 30 June, 2014; accepted 30 June, 2014; published online 31 July, 2014

Keywords: epithelial-mesenchymal transition, Hertwig's epithelial root sheath/epithelial rests of Malassez cells, immortalization, stemness, SV40 large T antigen

\section{INTRODUCTION}

Hertwig's epithelial root sheath (HERS) from the enamel organ has a crucial role in root formation in teeth (Spouge, 1980). HERS cells remain in the periodontium as epithelial rests of Malassez (ERM) after tooth development (Wentz et al., 1950), and ERM could have a functional role in cementum or enamel repair/regeneration (Foster et al., 2007; Hasegawa et al., 2003; Shinmura et al., 2008). In adult teeth, these HERS/ERM cells are a unique epithelial cell population and are reported to have stem cell characteristics (Nam et al., 2011; Xiong et al., 2012). However, more studies are required to understand the exact roles of HERS/ERM cells.

Primary cells cannot indefinitely expand in vitro. This limitation can be overcome through immortalization using the ectopic expression of oncogenes, which surpass cell cycle arrest. The viral oncogene SV40 large T antigen (SV40 LT) can inhibit senescence and induces immortalization (Bryan and Reddel, 1994). SV40 LT inactivates the tumor suppressor p53 and Rb pathways, leading to cell growth extension (Shay et al., 1991; Wright et al., 1989). HERS/ERM cells comprise a very small population and appear to be in a quiescent state (Shinmura et al., 2008). Thus, it has been difficult to expand primarily isolated HERS/ERM cells in vitro. In this study, we established immortalized HERS/ERM cells by overexpressing SV40 LT, and the immortalized HERS/ERM cells were maintained for more than 20 passages. The immunophenotypic characteristics of immortalized HERS/ERM cells were compared with those of primary HERS/ERM cells and showed characteristics of normal HERS/ ERM cells. The epithelial-mesenchymal transition (EMT) of immortalized HERS/ERM cells was induced via TGF- $\beta 1$. Finally, the stemness of the immortalized HERS/ERM cells was determined.

\section{MATERIALS AND METHODS}

Primary isolation and culture of human HERS/ERM cells Human third molars were obtained in Hank's balanced salt solution (HBSS) (Welgene, Korea) supplemented with 3\% Antibiotic-Antimycotic (Gibco, USA) at $4^{\circ} \mathrm{C}$. Periodontal ligament tissues were extracted with fine forceps and were minced and incubated in $1 \mathrm{mg} / \mathrm{ml}$ of Collagenase type I (Gibco) and $2.4 \mathrm{mg} /$ $\mathrm{ml}$ of Dispase (Gibco) at $37^{\circ} \mathrm{C}$ for $1 \mathrm{~h}$. To isolate the HERS/ 
ERM cells, after inactivating enzyme digestion by $10 \%$ FBS (HyClone, USA) in RPMI 1640 (Welgene, Korea), the cells were washed two times with serum-free keratinocyte basal medium (KBM) (Lonza, USA). Single-cell suspensions were maintained in serum-free keratinocyte growth medium (KGM) (Lonza) with provided supplement (Lonza) for $30 \mathrm{~min}$. The supernatant was then transferred to a new dish to culture the HERS/ERM cells. Before colonies of HERS/ERM cells were observed, half of the medium was replaced every 2 days. After the colonies were formed, whole amount of the medium was changed every 2 days. The cells were sub-cultured at $70 \%$ confluency. At each passage, cells were both counted and photographed, and the population doubling length (PDL) was calculated. To induce EMT, primary HERS/ERM cells and immortalized HERS/ERM cells were treated with $10 \mathrm{ng} / \mathrm{ml}$ of TGF- $\beta 1$ (Peprotech, USA) for $48 \mathrm{~h}$.

\section{SV40 LT transformation}

Primary HERS/ERM cells at passage 3 were used for electroporation following a previous report. Briefly, $20 \mu \mathrm{g}$ of pRNS-1 plasmid were transfected into $1 \times 10^{6} \mathrm{HERS} / \mathrm{ERM}$ cells via electroporation at $250 \mathrm{~V}$ and $500 \mu \mathrm{F}$ with a Gene Pulser (BioRad, USA). The transfection mixture was cultured for 2 days in fresh KGM and then G418 (Cellgro Mediatech, USA) was added to the medium at a concentration of $100 \mu \mathrm{g} / \mathrm{ml}$. The medium supplemented with G418 was changed every other day. Two weeks later, independent colonies were picked, transferred to new culture dishes, passaged and maintained with G418-free culture medium. At each passage, the cells were counted, and the population doubling length (PDL) was calculated.

\section{FACS analysis}

For fluorescence-activated cell sorter (FACS) analysis, the cells were detached and washed with PBS supplemented with $2 \%$ FBS. The following antibodies were used: FITC-conjugated mouse anti-human CD14, CD31, CD44, CD45, PE-conjugated mouse anti-human CD29, CD73, CD117, and APC-conjugated mouse anti-human CD34, CD105, and HLA-DR (all from BD Pharmingen). Between 100,000 and 500,000 cells were incubated with antibody for $30 \mathrm{~min}$ on ice. After washing, the cells were fixed with $4 \%$ paraformaldehyde at $4^{\circ} \mathrm{C}$ prior to analysis. The fluorescence intensity was measured on a FACS Calibur (Becton Dickinson, USA), and the data were analyzed using FLOWJO (Tree Star, Inc., USA) software.

\section{Quantitative PCR and semi-quantitative PCR}

Total RNA of cells was obtained from primary HERS/ERM cells and immortalized HERS/ERM cells before and after using an RNeasy Mini Kit (Qiagen, USA). The total RNA $(2 \mu \mathrm{g})$ was reverse-transcribed with M-MLV (Invitrogen TM, USA) and oligo dT during a $50 \mathrm{~min}$ incubation at $37^{\circ} \mathrm{C}$ followed by a 15 min incubation at $70^{\circ} \mathrm{C}$. Using resulting CDNA as the template, quantitative PCR and semi-quantitative PCR was performed with specific primers for the target genes (Supplementary Table 1). Quantitative PCR was performed using a CFX Connect Real-Time PCR Detection System (Bio-rad). The cDNA were amplified in a reaction mixture $(20 \mu \mathrm{l})$ containing $10 \mu \mathrm{l}$ of THUNDERBIRD SYBR qPCR Mix (QPS-201, TOYOBO, Japan) and $0.25 \mu \mathrm{M}$ of each primer. Glyceraldehyde-3-phosphate dehydrogenase (GAPDH) was used as internal normalization controls for mRNAs. All the samples were analyzed in duplicates.

Semi-quantitative PCR was performed with i-MAXII (Intron, Korea). The conditions of PCR used for the amplification of the epithelial stem cell-related genes ( $A B C G 2, E p C A M, B m i-1$, and $\triangle N p 63)$ and the embryonic stem cell-related genes (Oct-4, Sox2, and Nanog) have been previously described (Nam and Lee, 2009; Nam et al., 2011). The PCR products were separated on $1.5 \%$ agarose gel containing ethidium bromide.

\section{RESULTS}

Immortalization of HERS/ERM cells via the overexpression of SV40 LT

HERS/ERM cells are considered to be quiescent and have limited lifespans (Shinmura et al., 2008). SV40 LT was transfected into isolated primary HERS/ERM cells via electroporation. Four clones were established via G418 selection for 14 days. Within four clones, one clone was chosen and investigated during long-term culture because this clone could be maintained for more than 20 passages. However, primary HERS/ERM cells could not proliferate for more than 6 passages (Fig. 1B). There were no morphological differences between the primary HERS/ERM cells and immortalized HERS/ERM cell line (Fig. 1A).

\section{Immunophenotype}

To compare immunophenotypes between primary HERS/ERM cells and the immortalized HERS/ERM cell line, a FACS analysis was performed. Primary HERS/ERM cells were positive for some mesenchymal cell markers, such as CD29 and CD73, but negative for hematopoietic cell markers (CD14, CD34, CD117, HLA-DR) and endothelial cell markers (CD31) (Fig. 2A). However, the expression of CD10, CD44, CD90, and CD105 was low. Immortalized HERS/ERM cells showed similar surface marker expression patterns (Fig. 2B). These data suggested that the immunophenotypic characteristics of the immortalized HERS/ERM cells did not differ from those of the primary HERS/ERM cells.

\section{Epithelial-mesenchymal transition (EMT)}

In a previous report, primary HERS/ERM cells could acquire a mesenchymal phenotype through the EMT via TGF- $\beta 1$ (Sonoyama et al., 2007). To investigate the EMT of immortalized HERS/ERM cells, the cells were treated with TGF- $\beta 1$ for $48 \mathrm{~h}$. TGF- $\beta 1$ induced morphological changes in primary HERS/ERM cells and immortalized HERS/ERM cells, such as an elongated shape and a loss of cell-cell contact (Figs. 3A and 3B). To confirm EMT, the expression of E-cadherin, N-cadherin, and vimentin was examined. As shown in Fig. 3C, after EMT, primary and immortalized HERS/ERM cells demonstrated the upregulated expression of $\mathrm{N}$-cadherin and vimentin. However, the expression of E-cadherin underwent no significant change. These data suggested that immortalized HERS/ERM cells could respond to TGF- $\beta 1$ and acquire mesenchymal phenotypes through EMT.

\section{Stemness in immortalized HERS/ERM cells}

Previously, we reported that HERS/ERM cells expressed embryonic stem cell (ESC) and epithelial stem cell (EpiSC) markers (Nam et al., 2011). As shown Fig. 4A, primary HERS/ERM cells expressed EpiSC markers (ABCG2, Bmi-1, p63) and ESC markers (Oct-4, Nanog, Sox-2). Similar to primary HERS/ERM cells, immortalized HERS/ERM cells showed EpiSC markers and ESC markers, although the expression levels were slightly different. These data suggested that immortalized HERS/ERM cells maintained stem cell characteristics that were comparable with those of primary HERS/ERM cells. 


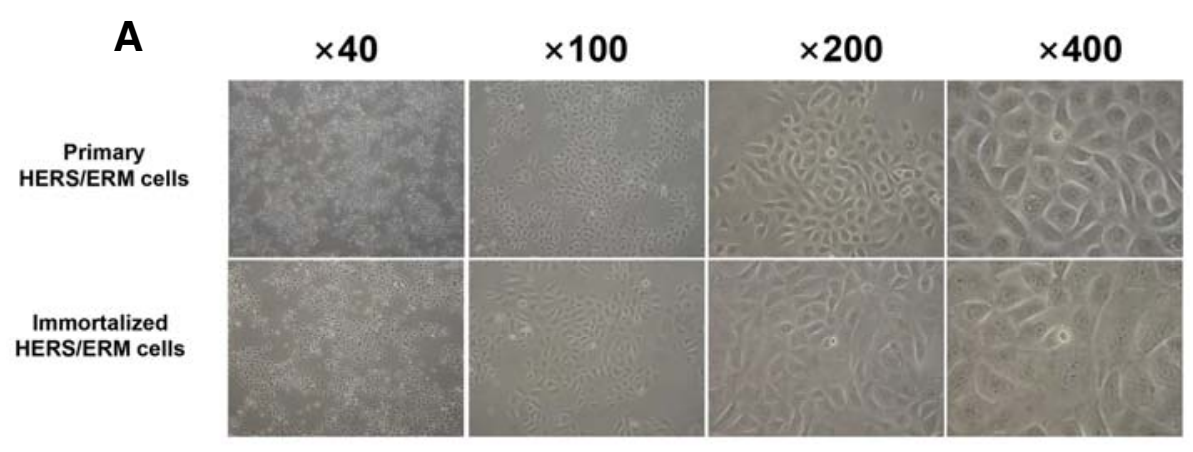

B

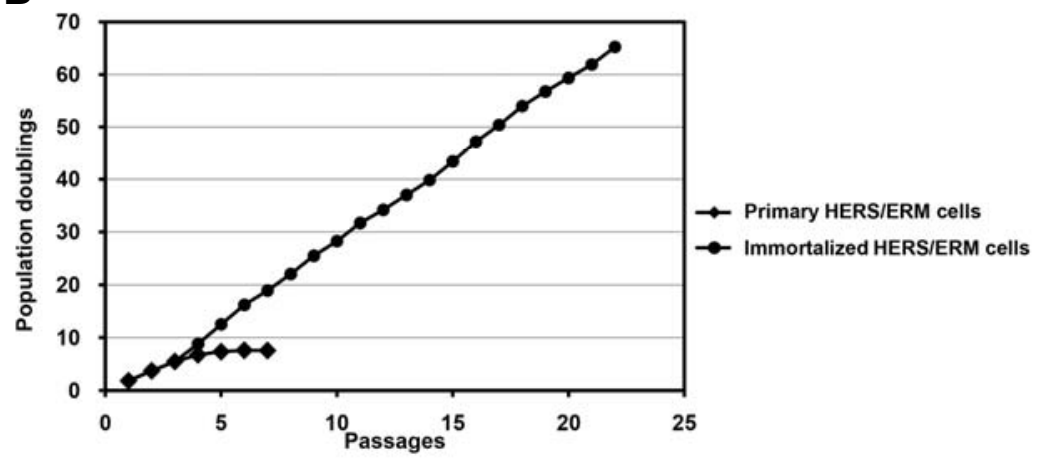

Fig. 1. Characterization of primary HERS/ERM cells and immortalized HERS/ERM cells. (A) Primary HERS/ ERM cells showed a typical epithelial cell morphology. Compared with primary HERS/ERM cells, no morphological differences were observed in the immortalized HERS/ ERM cells. (B) Primary HERS/ERM cells could not be maintained for more than 6 passages. However, immortalized HERS/ERM cells expanded for more than 20 passages. Moreover, no growth regression was detected during the late passages.
A

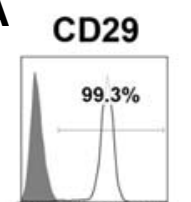

\section{CD14}

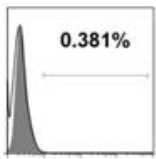

B

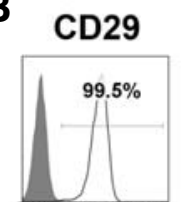

CD14

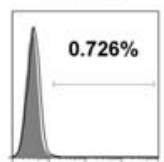

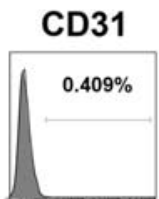

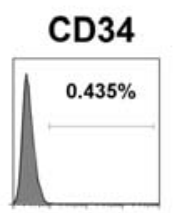

CD31

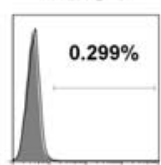

CD34

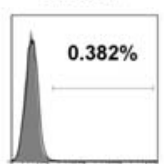

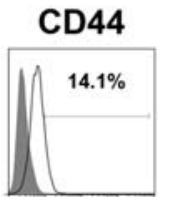
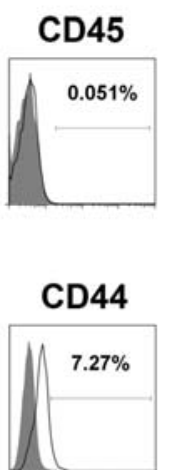

CD45

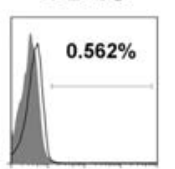

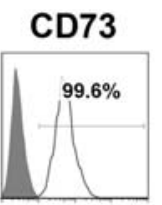

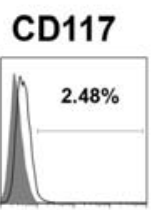

CD73

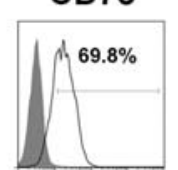

CD117

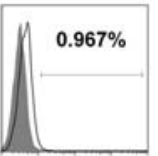

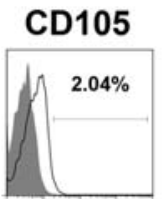

HLA-DR
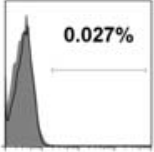

\section{CD105}

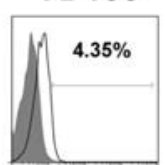

HLA-DR

$0.230 \%$

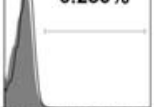

Fig. 2. Immunophenotypes of primary HERS/ ERM cells and immortalized HERS/ERM cells. To determine the immunophenotypes of primary HERS/ERM cells (A) and immortalized HERS/ ERM cells (B), the expression of mesenchymal, endothelial, and hematopoietic markers was analyzed via flow cytometry. The expression of CD44 and CD105 was weakly detected in primary HERS/ERM cells and immortalized HERS/ ERM cells. Endothelial and hematopoietic markers were not detected.

\section{DISCUSSION}

Stem cells represent a useful resource for regenerative medicine. Because most organs contain tissue-specific stem cells, the isolation, characterization, and ex vivo expansion of resi- dent stem cells are necessary for tissue engineering. However, HERS/ERM cells have been difficult to sufficiently expand for in vitro characterization and in vivo transplantation. To overcome these limitations, we established SV40 LT-immortalized HERS/ ERM cells. To our knowledge, this work is the first report on the 
A

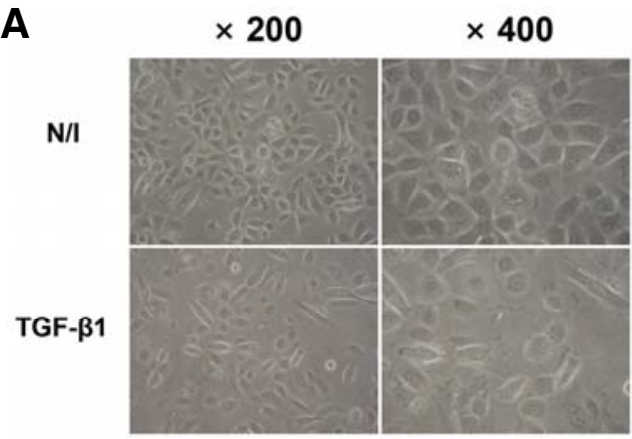

B

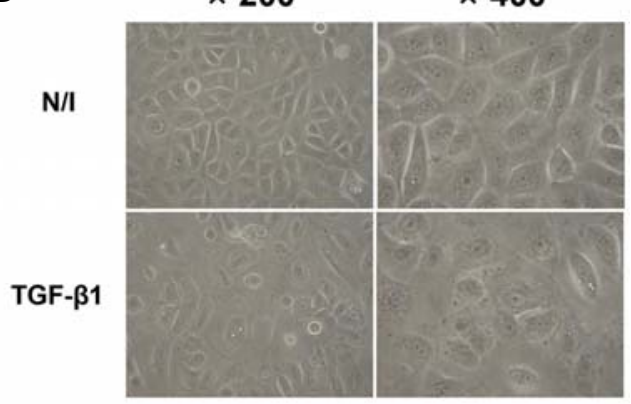

C

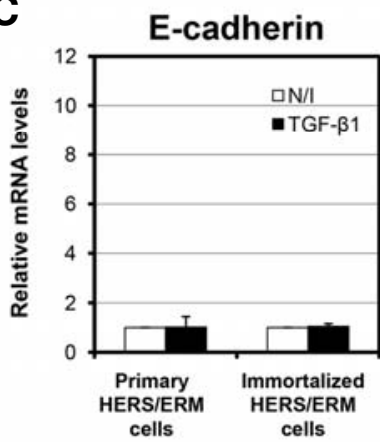

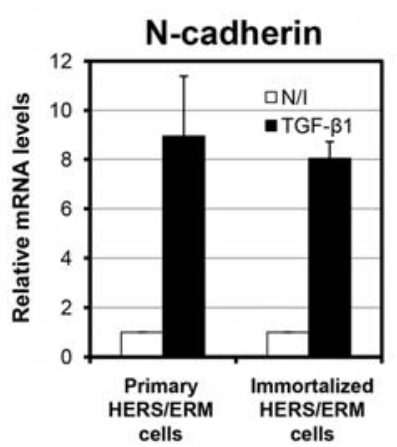

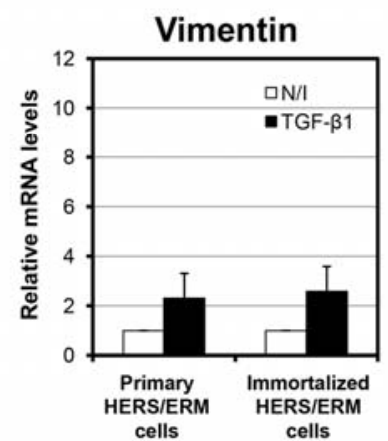

Fig. 3. Epithelial-mesenchymal transition (EMT) of primary HERS/ERM cells and immortalized HERS/ERM cells. The EMT of primary HERS/ERM cells and immortalized HERS/ERM cells was induced via TGF- $\beta 1$. (A) After $48 \mathrm{~h}$, TGF- $\beta 1$-treated primary HERS/ERM cells showed elongated morphology and loose cell-to-cell contact. (B) Compared with primary HERS/ERM cells, similar morphological changes were observed in immortalized HERS/ERM cells after TGF- $\beta 1$ treatment. (C) To confirm EMT, the expression of E-cadherin, N-cadherin, and vimentin was determined using quantitative PCR. When TGF- $\beta 1$ was treated, the expression of N-cadherin and vimentin was increased in both the primary HERS/ERM and immortalized HERS/ERM cells. However, there were no significant changes in the expression of E-cadherin. The data were expressed as the means \pm SD.

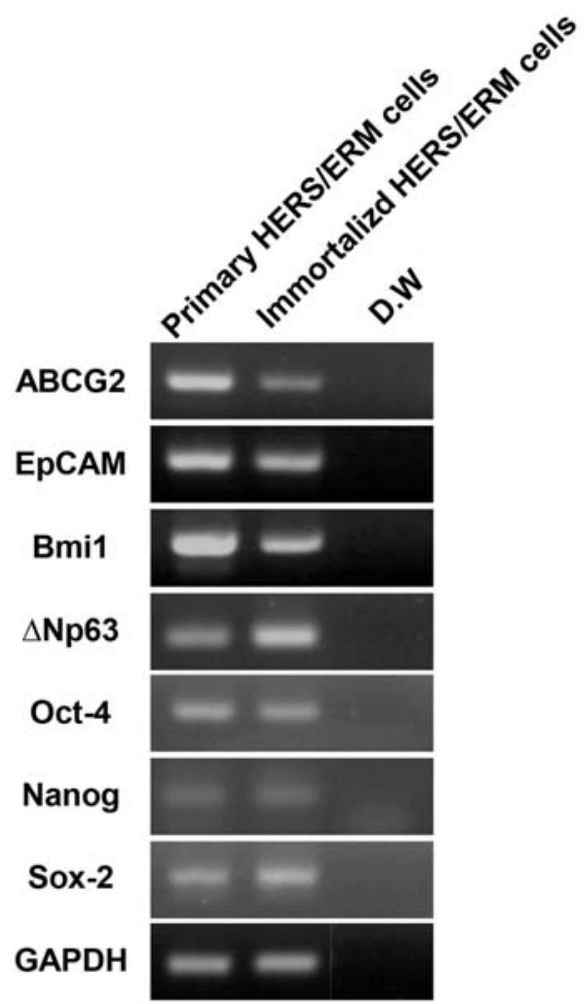

Fig. 4. Stemness of primary HERS/ERM cells and immortalized HERS/ERM cells. The expression pattern of epithelial stem cell (EpiSC) and embryonic stem cell (ESC) markers was compared in primary HERS/ERM cells and immortalized HERS/ERM cells. RT-PCR data showed no significant expression differences between EpiSC and ESC markers in both cell types. 
immortalization of human HERS/ERM cells.

Human periodontal ligament contains various types of cells, including PDLSCs and HERS/ERM cells. The reciprocal interactions between PDLSCs and HERS/ERM cells could contribute to the homeostasis of the periodontium. To better understand HERS/ERM cells, primary isolation and in vitro expansion of HERS/ERM cells are the first step. Because the amount of periodontium is small and HERS/ERM cells are known to be in a quiescent state, the primary isolation of HERS/ERM cells has been difficult. To overcome these problems, SV40 LT was used to immortalize various mammalian cells, including mouse HERS/ERM cells (Zeichner-David et al., 2003). SV40 LT antigen suppresses p53 and cell cycle arrest and apoptosis (Chen and Hahn, 2003). More recently, p53-deficient mice were used to culture dental epithelial cells (Komine et al., 2007; Takahashi et al., 2010). To overcome the limited lifespan of human HERS/ ERM cells, we used the pRNS-1 plasmid, which contains oriSV40 LT and a neomycin resistance cassette. We obtained four clones after G418 selection. Interestingly, the four clones showed different morphological and proliferative characteristics (data not shown). This may suggest that the HERS/ERM cells were heterogeneous. Finally, one clone was chosen, which showed a higher proliferation rate and a morphology that was most similar to that of primary HERS/ERM cells. After long-term culture, immortalized HERS/ERM cells did not show altered morphology or immunophenotypes compared with primary HERS/ERM cells.

EMT is involved in embryonic development, wound healing, fibrosis, and tumor metastasis (Thiery et al., 2009). There has been controversy over the EMT of HERS/ERM cells. Because HERS/ERM cells represent a heterogeneous population, not all HERS/ERM cells have the ability to undergo EMT (Akimoto et al., 2011; Zeichner-David et al., 2003). Recently, EMT was demonstrated to be one mechanism by which HERS/ERM cells generate cementum (Sonoyama et al., 2007; Xiong et al., 2012). The responsiveness to TGF- $\beta 1$ could be an important indicator of functional HERS/ERM cells. With this knowledge, we investigated the responsiveness of immortalized HERS/ERM cells to TGF- $\beta 1$. After treatment with TGF- $\beta 1$, immortalized HERS/ ERM cells showed an increased expression of $\mathrm{N}$-cadherin and vimentin, which are well known mesenchymal markers. Unexpectedly, the expression of E-cadherin was not decreased in either the primary HERS/ERM cells or immortalized HERS/ ERM cells. Breast carcinoma cell lines showed no change in Ecadherin expression after EMT, which can be explained by reversible EMT or incomplete EMT (Chao et al., 2010; Jo et al., 2009). To identify EMT or reverse the EMT of HERS/ERM cells, more studies, such as one on longer TGF- $\beta 1$ treatment, are necessary.

Stem cells reside in adult organs and regenerate their own tissues during adult life. Recently, the overexpression of Oct-4, Sox-2, KIf-4, and c-Myc was shown to reprogram somatic cells and induce a pluripotent state (Takahashi et al., 2007). This groundbreaking study suggested that the expression of pluripotent genes could induce the stemness of adult stem cells. Recently, we, in addition to others, reported that HERS/ERM cells contain primitive stem cells, which express not only epithelial stem cell but also embryonic stem cell markers (Nam and Lee, 2009; Nam et al., 2011). In our results, HERS/ERM cells demonstrated the expression of $A B C G 2, E p C A M, B m i 1$, and p63, which are epithelial stem cell markers. Moreover, the expression of Oct-4, Nanog, and Sox-2, which are embryonic stem cell markers, confirmed the maintenance of primitive characteristics after the immortalization of HERS/ERM cells.
In this study, we demonstrated the usefulness of SV40 LT in immortalizing HERS/ERM cells. With the exception of the overexpression of SV40 LT, HERS/ERM cells had similar characteristics to primary HERS/ERM cells. HERS/ERM cells are considered to be a unique source for dental epithelial cells resident in the human periodontium. We expect immortalized HERS/ERM cells to contribute to the understanding of biological functions of HERS/ERM cells and regenerative medicine of the periodontium.

Note: Supplementary information is available on the Molecules and Cells website (www.molcells.org).

\section{ACKNOWLEDGMENTS}

This research was supported by a grant from National Research Foundation (NRF-2011-0010100) and the Korean Health Technology R\&D Project, Ministry of Health \& Welfare, Republic of Korea (A110911).

\section{REFERENCES}

Akimoto, T., Fujiwara, N., Kagiya, T., Otsu, K., Ishizeki, K., and Harada, H. (2011). Establishment of Hertwig's epithelial root sheath cell line from cells involved in epithelial-mesenchymal transition. Biochem. Biophys. Res. Commun. 404, 308-312.

Bryan, T.M., and Reddel, R.R. (1994). SV40-induced immortalization of human cells. Crit. Rev. Oncog. 5, 331-357.

Chao, Y.L., Shepard, C.R., and Wells, A. (2010). Breast carcinoma cells re-express E-cadherin during mesenchymal to epithelial reverting transition. Mol. Cancer 9, 179 .

Chen, W., and Hahn, W.C. (2003). SV40 early region oncoproteins and human cell transformation. Histol. Histopathol. 18, 541-550.

Foster, B.L., Popowics, T.E., Fong, H.K., and Somerman, M.J. (2007) Advances in defining regulators of cementum development and periodontal regeneration. Curr. Top. Dev. Biol. 78, 47-126.

Hasegawa, N., Kawaguchi, H., Ogawa, T., Uchida, T., and Kurihara $\mathrm{H}$. (2003). Immunohistochemical characteristics of epithelial cell rests of Malassez during cementum repair. J. Periodontal. Res. 38, 51-56.

Jo, M., Lester, R.D., Montel, V., Eastman, B., Takimoto, S., and Gonias, S.L. (2009). Reversibility of epithelial-mesenchymal transition (EMT) induced in breast cancer cells by activation of urokinase receptor-dependent cell signaling. J. Biol. Chem. 284, 22825-22833.

Komine, A., Suenaga, M., Nakao, K., Tsuji, T., and Tomooka, Y. (2007). Tooth regeneration from newly established cell lines from a molar tooth germ epithelium. Biochem. Biophys. Res. Commun. 355, 758-763.

Nam, H., and Lee, G. (2009). Identification of novel epithelial stem cell-like cells in human deciduous dental pulp. Biochem. Biophys. Res. Commun. 386, 135-139.

Nam, H., Kim, J., Park, J., Park, J.C., Kim, J. W., Seo, B.M., Lee, J.C., and Lee, G. (2011). Expression profile of the stem cell markers in human Hertwig's epithelial root sheath/Epithelial rests of Malassez cells. Mol. Cells 31, 355-360.

Shay, J.W., Wright, W.E., and Werbin, H. (1991). Defining the molecular mechanisms of human cell immortalization. Biochim. Biophys. Acta 1072, 1-7.

Shinmura, Y., Tsuchiya, S., Hata, K., and Honda, M.J. (2008). Quiescent epithelial cell rests of Malassez can differentiate into ameloblast-like cells. J. Cell. Physiol. 217, 728-738.

Sonoyama, W., Seo, B.M., Yamaza, T., and Shi, S. (2007). Human Hertwig's epithelial root sheath cells play crucial roles in cementum formation. J. Dent. Res. 86, 594-599.

Spouge, J.D. (1980). A new look at the rests of Malassez. A review of their embryological origin, anatomy, and possible role in periodontal health and disease. J. Periodontol. 51, 437-444

Takahashi, K., Tanabe, K., Ohnuki, M., Narita, M., Ichisaka, T., Tomoda, K., and Yamanaka, S. (2007). Induction of pluripotent stem cells from adult human fibroblasts by defined factors. Cell 131, 861-872.

Takahashi, C., Yoshida, H., Komine, A., Nakao, K., Tsuji, T., and 
Tomooka, Y. (2010). Newly established cell lines from mouse oral epithelium regenerate teeth when combined with dental mesenchyme. In Vitro Cell. Dev. Biol. Anim. 46, 457-468.

Thiery, J.P., Acloque, H., Huang, R.Y., and Nieto, M.A. (2009). Epithelial-mesenchymal transitions in development and disease. Cell 139, 871-890.

Wentz, F.M., Weinmann, J.P., and Schour, I. (1950). The prevalence, distribution, and morphologic changes of the epithelial remnants in the molar region of the rat. J. Dent. Res. 29, 637646.

Wright, W.E., Pereira-Smith, O.M., and Shay, J.W. (1989). Reversi- ble cellular senescence: implications for immortalization of normal human diploid fibroblasts. Mol. Cell. Biol. 9, 3088-3092.

Xiong, J., Mrozik, K., Gronthos, S., and Bartold, P.M. (2012). Epithelial cell rests of Malassez contain unique stem cell populations capable of undergoing epithelial-mesenchymal transition. Stem Cells Dev. 21, 2012-2025

Zeichner-David, M., Oishi, K., Su, Z., Zakartchenko, V., Chen, L.S., Arzate, H., and Bringas, P., Jr. (2003). Role of Hertwig's epithelial root sheath cells in tooth root development. Dev. Dyn. 228, 651-663. 